\title{
In Vitro Assessment of the Neuroprotective and Antioxidant Properties of New Benzimidazole Derivatives as Potential Drug Candidates for the Treatment of Parkinson's Disease ${ }^{\dagger}$
}

\author{
Neda Anastassova 1,*, Maria Argirova 2, Denitsa Yancheva 3, Denitsa Aluani 3, Virginia Tzankova ${ }^{3}$, \\ Nadya Hristova-Avakumova ${ }^{3}$ and Vera Hadjimitova ${ }^{3}$ \\ 1 Institute of Organic Chemistry with Centre of Phytochemistry, Bulgarian Academy of Sciences, \\ 1113 Sofia, Bulgaria \\ 2 Department of Pharmacology, Faculty of Pharmacy, Medical University-Sofia, 1000 Sofia, Bulgaria \\ 3 Department of Medical Physics and Biophysics, Faculty of Medicine, Medical University-Sofia, \\ 1431 Sofia, Bulgaria \\ * Correspondence: neda@orgchm.bas.bg; Tel.: +359-2-9606106. \\ + Presented at the 2nd Molecules Medicinal Chemistry Symposium (MMCS): Facing Novel Challenges in \\ Drug Discovery, Barcelona, Spain, 15-17 May 2019.
}

Published: 8 August 2019

Keywords: neurodegenerative disorders; benzimidazoles; antioxidants

Oxidative stress is related to the pathogenesis of many neurodegenerative disorders, including Parkinson's and Alzheimer's disease. The inability of the neuronal cells to maintain redox balance leads to free radicals accumulation, mitochondrial dysfunction, and neuronal injury.

The neurons are highly sensitive to oxidative stress due to stronger dependence on oxidative phosphorylation, exposure to high concentrations of oxygen, and accumulation of metal ions during aging which increase the generation of reactive oxygen species. Other factors are the presence of easily oxidized polyunsaturated fatty acids and the relatively poor concentrations of antioxidants.

A series of new benzimidazole hydrazones containing hydroxy and methoxy substituents were synthesized as analogues of Melatonin-a known antioxidant with neuroprotective action. The neurotoxicological potential of the compounds was assessed, and the derivatives demonstrating the most prominent effects were studied for neuroprotective properties in different in vitro models: $\mathrm{H}_{2} \mathrm{O}_{2}$-induced oxidative stress in neuroblastoma SH-SY5Y cells and 6-hydroxydopamine (6-OHDA) induced neurotoxicity in rat brain synaptosomes. As markers of oxidative damage, SH-SY5Y cell viability, synaptosomal viability, and intra-synaptosomal content of GSH were used.

For further investigation of antioxidant properties, in vitro spectrophotometric model systems have been used. The antiradical activity against the stable free radicals ABTS and DPPH has been estimated, as well as the capability of the derivatives to decrease the level of molecular damage of biologically important molecules upon ferrous iron-induced oxidative molecular damage. The obtained data revealed that the tested compounds demonstrate a protective effect and capability to decrease the concentration of stable free radicals. Their potency depends of the used radical, oxidisable substrate, the type, and the position of the structural modification in the evaluated molecular structure.

Different possible mechanisms, such as hydrogen atom transfer (HAT), single-electron transfer (SET-PT), and sequential proton loss electron transfer (SPLET), were studied by DFT methods. 
Acknowledgments: The financial support by the National Science Fund of Bulgaria (contract No. КП-06-M29/4) is gratefully acknowledged.

(C) 2019 by the authors. Licensee MDPI, Basel, Switzerland. This article is an open access article distributed under the terms and conditions of the Creative Commons Attribution (CC BY) license (http://creativecommons.org/licenses/by/4.0/). 